\title{
EUTOMIA
}

Revista de Literatura e Linguística

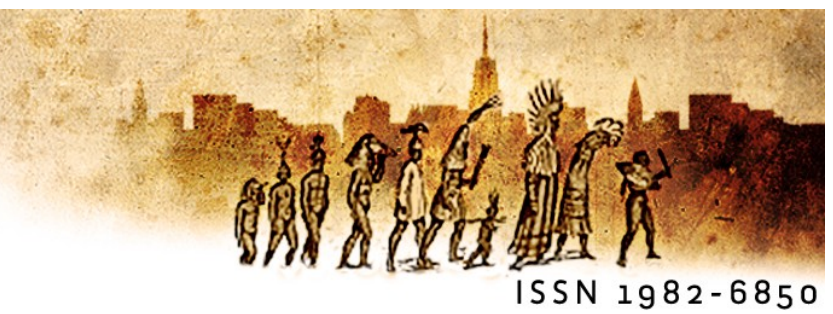

\section{La discussion du langage intérieur par L.S. Vygotskij}

\author{
Janette Friedrichi (Université de Genève)
}

\begin{abstract}
Résumé:
Dans ce texte est présenté la manière dont Lev S. Vygotskij participe au début du $20^{\mathrm{e}}$ siècle à la discussion autour du langage intérieur qui avait eu lieu en psychologie et en linguistique. II sera montré que c'est notamment l'idée d'une diversité fonctionnelle du langage défendue et développée par Lev P. Jakubinskij que Vygotskij utilise pour ses propres recherches. Le panorama que Vygotskij donne de ces débats nous servira de point de départ pour esquisser la démarche proposée par lui pour s'attaquer au problème que le langage intérieur pose à une recherche qui veut mettre à jour sa nature psychologique.
\end{abstract}

Mots-clés: Langage intérieur; psycho-génétique; sémiotico-descriptive.

\section{Resumo:}

Neste texto é apresentada a maneira como Lev S. Vygotskij participava, no início do século $X X$, da discussão sobre linguagem interior no espaço da psicologia e da linguística. Mostrará que foi, notadamente, a ideia de uma diversidade funcional da linguagem defendida e desenvolvida por Lev $P$. Jakubinskij que Vygotskij utilizou para as suas próprias pesquisas. $O$ panorama desses debates, traçado por Vygotskij, nos servira como ponto de partida para delinear o encaminhamento proposto por ele próprio para enfrentar o problema que a linguagem põe em evidência para uma pesquisa que quer atualizar sua própria psicologia.

Palavras-chave: Linguagem interior; psicogenética; semiótico-descritiva.

\footnotetext{
1 Este artigo foi publicado primeiramente em Langue Française, vol.132, dezembro 2001, p. 57-71: <http://www.persee.fr/doc/lfr_0023-8368_2001_num_132_1_6315> e com publicação autorizada pela autora para a Revista Eutomia. A formatação do presente artigo teve que ser modificada para atender às normas da ABNT e da revista. Ele faz parte de um número temático: La parole intérieure, organizado por Gabriel Bergounioux. Acrescentamos a literatura mais atualizada sobre esse tema.
} 
Si les analyses sur le langage intérieur ont connu au cours du vingtième siècle une certaine éclipse dans la linguistique, en psychologie l'intérêt pour ce phénomène n'a jamais vraiment cessé. C'est, en psychologie, chez les chercheurs s'inscrivant dans le courant socio-culturel qu'on trouve le plus d'allusions, de discussions et de recherches autour du langage intérieur ; cela n'a du reste rien d'étonnant puisque l'un des fondateurs de ce courant, L.S. Vygotskij (1896 -1934), propose en 1934 dans le dernier chapitre de son livre "Pensée et langage" une analyse originale du langage intérieur. Les références aux différentes conceptions du langage intérieur toutes développées dans les années 20 du vingtième siècle que Vygotskij fait dans ce chapitre forcent néanmoins à déplorer le peu d'intérêt porté aujourd'hui à cette forme langagière. Bien que sommaire le panorama donné par Vygotskij des débats dans la psychologie de son temps permet de distinguer trois manières d'utiliser les termes "langage intérieur" ou endophasie. Ce panorama nous servira de point de départ pour esquisser la démarche proposée par Vygotskij pour s'attaquer au problème que le langage intérieur pose à une recherche qui veut mettre au jour sa nature psychologique.

Vygotskij montre que le terme "langage intérieur" est utilisé pour désigner des phénomènes bien différents. Dans une première acception, le langage intérieur est comparé à la mémoire verbale et plus spécifiquement à la reproduction des mots dans la mémoire, ce qui a comme résultat d'identifier les mots avec des idées, des représentations, des images. Cette démarche se rapproche d'une analyse purement psychologique des différentes étapes et mécanismes de remémorisation des mots attestable chez l'être humain. La deuxième manière de comprendre le langage intérieur est liée au courant béhavioriste et demeure, selon Vygotskij, dans le cadre de la pensée du sens commun. La considération du langage intérieur comme un langage moins les sons, comme un langage insonore, muet ou comme "un réflexe verbal inhibé dans sa partie motrice " (Bechterew) semble être la définition la plus pertinente pour un phénomène auquel l'accès direct n'est pas possible. Lorsqu'on admet, à I'instar des représentants du béhaviorisme, que l'aspect comportemental (matériel) est le seul objet à prendre en considération dans une analyse à caractère psychologique, les phénomènes supposés intérieurs ne peuvent être inférés qu'à partir 
de phénomènes attestables à l'extérieur. La troisième définition du terme en question que Vygotskij trouve chez K. Goldstein associe "au concept de langage intérieur l'aspect interne global de toute activité verbale" (VYGOTSKIJ, 1997, p. 441). Cette acception se réfère en effet à une expérience à caractère interne faite par tous les locuteurs qui réalisent une activité langagière. Cette expérience interne se dérobe à n'importe quelle analyse objective tout en étant considérée comme une condition nécessaire pour l'utilisation du langage. Selon Vygotskij, ce troisième emploi du terme désigne moins une activité langagière qu'une activité mentale ou affective-volitive: "Dans le meilleur des cas il englobe sous une forme indifférenciée tous les processus internes précédant l'acte de parler, c'est-à-dire tout l'aspect interne du langage extériorisé." (ibid. p. 442).

En opposition à ces trois acceptions du terme, Vygotskij affirme:

Une compréhension correcte du langage intérieur doit partir de l'idée que celui-ci est une formation d'une nature psychologique particulière, un type particulier d'activité verbale, ayant des caractéristiques absolument spécifiques et un rapport complexe avec les autres types d'activité verbal. (ibid.)

Deux premiers renseignements peuvent être retenus de cette affirmation. Premièrement, le langage intérieur représente pour l'auteur un type particulier d'action verbale qui n'est pas à confondre avec une action mentale. Longtemps considéré comme un objet privilégié de la psychologie, laquelle fait écho à une tradition philosophique pour laquelle la pensée est un discours logiquement articulé que l'esprit se tient à lui-même (PANACCIO, 1999), Vygotskij introduit ce phénomène dans le domaine des investigations linguistiques. Deuxièmement, le langage intérieur n'est appréhendé ni comme ce qui précède le langage extériorisé et s'achève en lui ni comme ce qui reproduit le langage extériorisé dans la mémoire, mais comme une activité verbale absolument spécifique. Dans le même paragraphe, Vygotskij donne deux définitions, une pour le langage extériorisé, l'autre pour le langage intérieur qui vont toutes deux déterminer sa démarche ultérieure :

Le langage extériorisé est un processus de transformation de la pensée en paroles, sa matérialisation, son objectivation. Le langage intérieur est un processus de sens inverse, qui va de l'extérieur à l'intérieur, un processus de volatilisation du langage dans la pensée. (VYGOTSKIJ, 1997, p. 442-443). 
II serait faux d'en conclure que le langage ne joue guère de rôle dans le processus de pensée. Bien au contraire, le processus de volatilisation du langage demande, pour être réalisé, que le sujet pensant se serve du langage. Cela a comme conséquence que le langage acquiert des caractéristiques linguistiques bien spécifiques qui lui permettent de remplir cette fonction. C'est pourquoi Vygotskij ne cesse de souligner l'idée que le langage intérieur est une activité verbale tout à fait spécifique et originale aussi bien dans son fonctionnement que dans sa structure. Cette idée est souvent reprise et discutée dans les recherches actuelles sur l'œuvre de Vygotskij, mais les interprétations qui ont été développées concernant le langage intérieur emportent cependant une certaine ambiguïté. La démarche générale que nous suivrons consiste à préciser en quoi consiste cette ambiguïté et à tirer parti de cet éclaircissement pour proposer une lecture un peu différente de la manière dont Vygotskij discute le langage intérieur.

\section{Une lecture à caractère psycho-génétique}

Sans vouloir résumer toutes les prises de position à ce sujet, nous croyons justifié de constater que dans la littérature qui s'intéresse à l'œuvre de Vygotskij, une lecture à caractère psycho-génétique est prépondérante. Quatre exemples doivent suffire pour illustrer ce type d'interprétation. Le premier est tiré du dictionnaire de la psychologie dans lequel J.-E. Gombert écrit que le terme langage intérieur "désigne l'imagerie verbale en œuvre dans l'activité de pensée. Cette évocation verbale interne, manifeste lors de la lecture silencieuse ou de l'écriture, est plus généralement le langage de la réflexion. L.S. Vygotskij en fait un enjeu majeur du développement intellectuel: le langage, initialement communicatif, évolverait dans le sens d'une possibilité de plus en plus importante d'intériorisation" (DORON \& PAROT, 1991, p. 413). Il semble que cette compréhension du langage intérieur comme "évocation verbale interne" coïncide avec la première mais également avec la troisième définition critiquée par Vygotskij. Nous allons revenir un peu plus loin sur la fonction de réflexion que Gompert attribue au langage intérieur. Signalons que le recours au concept d'intériorisation place clairement le langage intérieur sur l'axe temporel de 
l'évolution orienté par le passage du langage extériorisé vers le langage intérieur. Une façon semblable d'aborder la relation entre le langage extériorisé et le langage intérieur est soutenue en linguistique en 1954 par R. Jakobson et a eu tendance à se généraliser dans les recherches ultérieures. Jakobson dit ceci :

Quant au discours non extériorisé, non prononcé, ce qu'on appelle le langage intérieur, ce n'est qu'un substitut elliptique et allusif du discours explicite et extériorisé. D'ailleurs, le dialogue sous-tend même le discours intérieur, comme l'ont démontré une série d'observations, de Peirce à Vygotsky. (JAKOBSON, 1963, p. 32).

La réticence vis-à-vis de toute autonomie et indépendance du langage intérieur par rapport au langage extériorisé exprimée dans les deux cas signale un glissement du sens du terme : le langage intérieur est en fait traité comme un sousproduit du langage extériorisé, ce qui pousse le chercheur à faire l'économie de la question quant à sa spécificité sur le plan psychologique et linguistique.

Les deux lectures suivantes proposent une analyse plus fine et rendent justice à l'idée vygotskienne d'une autonomie, voire même d'une opposition du langage intérieur par rapport au langage extériorisé. Dans le beau livre de $C$. Knobloch qui résume l'histoire récente de la psychologie du langage (Sprachpsychologie), I'auteur s'arrête en détail sur le changement de la fonction du langage attestable lorsque le langage social se transforme en langage intérieur. Knobloch souligne l'importance du langage intérieur pour la production des énoncés langagiers et pour leur compréhension puisqu'il présente "le mécanisme qui fournit au 'programme' grammatico-sémantique les matériaux quasi-langagiers sous une forme condensée, compacte et indicative" (KNOBLOCH, 1984, p. 230). En d'autres termes, Knobloch caractérise le langage intérieur une fois constitué comme une espèce de structure profonde, une structure antérieure au langage extériorisé permettant la production des phrases et des formes langagières attestables dans la communication et il se rapproche alors de la définition de Goldstein. C'est le langage intérieur qui, selon Knobloch, rend possible la construction des propositions hors contexte. Même si le langage intérieur est discuté à l'égard de sa fonction comme un type de langage relativement autonome par rapport au langage communicatif, Knobloch insiste néanmoins sur le lien génétique et actuel entre ces deux types de langage. Le lien se révèle comme génétique, car le langage intérieur est abordé comme un phénomène 
secondaire par rapport au langage extérieur, le premier étant le résultat de l'appropriation et de l'intériorisation du deuxième. Le lien est également considéré comme actuel, car il existe selon l'auteur un jeu réciproque entre ces deux langages qui donne lieu à l'émergence de la pensée. La production de la pensée se fait à travers deux mouvements, elle a lieu 1) dans la transformation du langage intérieur en un langage communicatif et compréhensible, et 2) dans la transformation du langage extérieur lors de son passage à l'intérieur. D'autres auteurs comme par exemple Rochex (1997) discutent la relation entre les deux types de langage également en termes d'unité et de discordance et leurs réflexions confirment ce qui est amorcé chez Knobloch: même si les deux mouvements cités ont une direction contraire, le langage joue dans les deux mouvements le rôle d'une médiation sémiotique. Dans le mouvement de l'intérieur vers l'extérieur le sens se modifie lorsqu'il trouve son incarnation dans les mots; dans celui de l'extérieur vers l'intérieur la pensée se développe au travers du langage intérieur. On pourrait en conclure que les auteurs intéressés par le langage intérieur dans l'œuvre de Vygotskij considèrent le langage dans la diversité de ses fonctions surtout comme moyen de médiation des processus cognitifs.

Ce qui est mis en lumière dans la discussion du langage intérieur, c'est en fait le processus d'intégration des phénomènes langagiers dans l'ensemble des fonctions psychiques et cognitives et les changements qu'une telle propagation du langagier suscite. Ainsi, le langage intérieur est considéré comme étant à la source d'importantes formations psychiques nouvelles: la capacité d'autoréflexion, la capacité de contrôler sa propre activité, d'entrer en contact social avec soi-même (Rochex) et la production des énoncés langagiers hors interaction (Knobloch) doivent leur existence au langage intérieur. L'importance que la théorie socio-culturelle accorde à l'idée de la contribution active des phénomènes langagiers à la constitution des moments internes du processus psychique trouve alors son reflet dans les interprétations que ces auteurs donnent du langage intérieur et que nous désignons comme "psycho-génétiques". L'adjectif "psychologique" souligne le fait que le langage intérieur est analysé dans sa fonction de médiatiser et par-là de développer les fonctions psychiques supérieures. L'adjectif "génétique" peut être compris dans 
deux sens. Tout d'abord il signale le fait que tous les auteurs cités traitent le langage intérieur comme provenant du langage extérieur en s'appuyant sur les analyses génétiques comme celles de Piaget, qui a démontré à l'aide du langage égocentrique que le langage extériorisé précède dans l'ontogenèse de l'enfant le langage intérieur. L'importance assignée par tous les auteurs au processus d'intériorisation trouve ici également son explication. Finalement, c'est exclusivement la nature sociale de la constitution du langage intérieur que Knobloch peut évoquer pour distinguer sa compréhension du langage intérieur de la conception chomskyenne des structures linguistiques profondes ${ }^{2}$.

Cela nous amène au deuxième emploi possible de l'adjectif "génétique ". Le langage intérieur une fois constitué est traité comme une condition quasi-causale des fonctions psychiques supérieures, notamment de la pensée. Rochex décrit ainsi le langage intérieur comme le domaine où le sens déborde celui du langage et il en conclut

aussi permet-il d'aborder aux rives de ce que Vygotskij nomme 'la sphère motivante de notre conscience', de cette 'tendance affective et volitive (qui) peut seule répondre au dernier pourquoi dans I'analyse de la pensée'"' (ROCHEX, 1997, p. 136).

Un peu plus loin, il cite encore une fois Vygotskij pour souligner l'idée d'une émergence causale de la pensée: "la pensée qui n'est pas motivée affectivement est impossible, comme le serait l'opération sans cause" (ibid.). L'interprétation du langage intérieur comme un moyen permettant d'accéder à la sphère motivante de la pensée rend toutefois le terme imprécis. S'agit-il d'une forme d'activité verbale bien spécifique et autonome qui attend en conséquence son analyse et sa description par des outils d'une linguistique moderne ou d'un phénomène duquel on peut inférer des causes primaires d'un mécanisme psychique supérieur? La deuxième manière de comprendre le langage intérieur trouve ses sources dans une approche omniprésente dans la pensée psychologique : I'idée que la conscience comme instance ultime dans

\footnotetext{
2 Signalons que les discussions autour du langage intérieur ont été dans les années 70 quasiment absorbées par les sciences cognitives qui cherchaient à prouver l'existence d'un langage de la pensée (angl. Language of thought). Par ce langage de la pensée ils entendent une structure propositionnelle ayant une caractère inné qui permettrait à expliquer la capacité des sujets se représenter mentalement le monde soit-il réel ou fictif, voir Fodor 1975. Les discussions sur le langage intérieur ont trouvé une autre continuation dans les recherches soviétiques qui mettaient au centre d'intérêt les conditions psychophysiologique du langage intérieur, voire Lurija 1982 ; Sokolov 1972.
} 
l'explication de la vie psychique est inséparablement liée à la recherche des moyens qui éveillent les faits psychiques au sein du psychisme (FRIEDRICH, 1993). En discutant le langage intérieur comme un tel "moyen", le terrain de la prise de conscience est prolongé jusque dans les sphères affectives et volitives. On pourrait en conclure que cette forme langagière est utilisée pour prouver la capacité du sujet à rendre réflexif la vie psychique dans sa complexité. Le problème est que le texte même de Vygotskij contribue à une telle compréhension du langage intérieur qui s'inscrit incontestablement dans le cadre d'une philosophie de la réflexion. Dans le dernier paragraphe de Pensée et langage qui clôt la discussion sur le langage intérieur, Vygotskij affirme que "La conscience se reflète dans le mot comme le soleil dans une petite goutte d'eau. Le mot est à la conscience ce qu'est un petit monde à un grand, ce qu'est une cellule vivante à l'organisme, un atome au cosmos" (VYGOTSKIJ, 1997, p. 500). Nous pensons cependant qu'une autre manière d'analyser le langage intérieur peut être dégagée de ce texte et proposons une lecture qui nous mènera à une compréhension de cette forme langagière un peu différente de celle discutée jusqu'à présent. Nous désignons cette nouvelle lecture comme sémiotico-descriptive en utilisant les mêmes termes que Bühler qui, en 1934, proposait d'ores et déjà dans sa "Théorie du langage " une distinction entre démarche psycho-génétique et démarche sémiotico-descriptive au sein de la linguistique (cf. BÜHLER, 1982, p. 287). Pour élucider la spécificité de la démarche sémiotico-descriptive un petit retour dans le contexte scientifique de l'union soviétique des années 20 est nécessaire.

\section{L'idée de la diversité fonctionnelle du langage}

La référence majeure du septième chapitre de Pensée et Langage est un livre peu connu en Europe occidentale: La parole dialogale, publié en 1923 par Lev P. Jakubinskij (1892-1945) ${ }^{3}$. Ce n'est que de loin en loin qu'on trouve des allusions dans la

\footnotetext{
${ }^{3}$ Voir la traduction d'extraits de ce livre par Archaimbault 2000. On peut affirmer que ce livre présente un texte clé pour la linguistique soviétique des années 20. Jakubinskij tente y d'articuler les idées de l'école linguistique de Saint-Petersbourg, dont il fait partie et le cercle linguistique de Moscou (voir par exemple les références de Jakubinskijs (1986 : 24) à Jakobson (1923)). Voir aussi les références que Bakhtine/Voloshinov (1977) font à Jakubinskij. Lev Jakubinskij, une linguistique de la parole (URSS, années 1920-1930), édition et traduction d'Irina Ivanova, en collaboration avec Patrick Sériot, Limoges : Lambert-Lucas, coll. « Bilingues en sciences humaines ».
} 
littérature à l'influence que Jakubinskij avait sur les réflexions de Vygotskij (cf. par exemple WERTSCH, 1988, chap.4). Cela est d'autant plus étonnant qu'on découvre que le septième chapitre de Pensée et langage est truffé d'énoncés et d'illustrations entières empruntés à Jakubinskij, même s'ils ne sont d'ailleurs pas souvent indiqués comme tels. Vygotskij constate ainsi que "(...) ces derniers temps le problème de la diversité fonctionnelle du langage est devenu l'une des premières préoccupations de la linguistique", et il poursuit en affirmant que "le langage n'est pas, même pour le linguiste, une forme unique d'activité verbale, c'est un ensemble de fonctions verbales variées" (VYGOTSKIJ, 1997, p. 468).

Dans une courte phrase qui suit, il explique qu'un tel examen du langage du point de vue fonctionnel consiste dans la prise en considération des conditions et du but de l'énoncé. Les conditions (psychologiques et sociologiques) et le but de l'énoncé seraient un des objets privilégiés des linguistes. Vygotskij répète ici avec d'autres mots ce que nous pouvons lire dans les trois premières pages du livre de Jakubinskij qui pose la " diversité fonctionnelle de la parole" comme objet ultime du linguiste.

A cette entreprise se joint un autre linguiste déjà connu à cette époque aussi bien en Europe occidentale qu'en Russie : R. Jakobson. Dans les thèses développées par le cercle linguistique de Prague pour le premier Congrès des philologues slaves en octobre 1929 à Prague (Thèses du C.L.P.) nous trouvons évoqué sous le point 3 les mêmes thèmes et les mêmes idées que ceux discutés par Jakubinskij. Ainsi, la thèse 3 est intitulée "Problèmes des recherches sur les langues de diverses fonctions" et le point a) porte le titre "Sur les fonctions de la langue". J. Fontaine (1974, p. 26) rapporte dans son ouvrage sur le cercle linguistique de Prague, que cette troisième thèse a bel et bien été préparée par Jakobson. A la différence de Jakubinskij, les auteurs des thèses ne parlent pas de la diversité fonctionnelle de la parole (des "formes fonctionnelles de la parole") mais des "Langues de diverses fonctions". Nous estimons cependant que les deux termes visent le même phénomène. Qu'entendent-ils par diversité fonctionnelle de la parole ou comme le dit Jakobson par langues de diverses fonctions? Jakubinskij se réfère à Humboldt qui en avait selon lui analysé deux d'entre elles : la prose et la poésie. L'argumentation de Jakubinskij est relativement facile à résumer: les deux diffèrent dans leur orientation et dans les outils 
linguistiques qu'elles utilisent. Chaque forme se sert de moyens langagiers bien différents de l'autre forme afin de les soumettre au but qui prédomine leur utilisation. En ce qui concerne par exemple la prose, Humboldt aurait montré que la langue de la prose scientifique possède une fonctionnalité qui consiste dans son adéquation au but, celui de présenter par ses caractéristiques langagières la logique qui corresponde au développement de la pensée scientifique. Pour atteindre ce but, elle utilise un lexique, une syntaxe et une grammaire tout à fait spécifiques et différents d'autres formes (voir JAKUBINSKIJ, 1986, p. 19-20). Vygotskij reprend ces idées et les résume d'une manière tout à fait analogue : "Dans l'une et l'autre forme de langage la langue a ses particularités qui se manifestent dans le choix des expressions, l'emploi des formes grammaticales et des procédés syntaxiques d'assemblage des mots" (VYGOTSKIJ, 1997, p. 469). La spécificité de chaque forme se perçoit donc dans ses particularités linguistiques; elle n'est pas à inférer des aspects extra-langagiers.

Dans les Thèses du C.L.P., Jakobson confirme et radicalise les affirmations de Jakubinskij. Même si sa terminologie est mal stabilisée et parfois même contradictoire, elle est parlante. II dit ceci :

Chaque langage fonctionnel a son système de conventions - la langue proprement dite ; il est par conséquent erroné d'identifier un langage fonctionnel avec la langue et un autre avec la 'parole' (dans la terminologie de Saussure), par ex. le langage intellectuel avec la 'langue' et le langage émotionnel avec la 'parole'. (THESES, 1929, p. 15).

Afin de dissiper le caractère imprécis de cette citation, nous proposons quelques éléments de clarification. Jakobson s'efforce visiblement d'exprimer ses divergences avec ce que les auteurs de thèses nomment eux-mêmes "la doxa saussurienne". En décrivant le langage fonctionnel comme un système de conventions, il met en question la distinction saussurienne entre la langue et la parole. Sans vouloir nous aventurer trop dans la discussion de savoir si les interprétations de Jakobson sont à la hauteur de l'œuvre de Saussure, il nous semble néanmoins justifié de dire que Jakobson renonce au concept de langue que Saussure définit entre autres comme un système ou un modèle qui sous-tiendrait ou expliquerait la prolifération quotidienne de la parole (cf. SAUSSURE, 1983, chap. 1-4). Pour Jakobson, la langue n'existe pas au singulier, ce qui ne veut pas non plus dire qu'il défend le point de vue 
"des langues naturelles", devenu important dans les lectures les plus récentes de l'œuvre de Saussure (cf. FEHR, 2000). Dans un certain sens, le point de départ de Jakobson est plus modeste et rompt entièrement avec les préoccupations de la linguistique historique. II s'agit d'une analyse des traits linguistiques spécifiques et à caractère conventionnel qui se montrent dans l'emploi du langage afin de réaliser différents buts. Le langage utilisé dans une fonction précise (réfléchir de manière scientifique, exprimer ses sentiments d'une manière poétique) possède des caractéristiques bien spécifiques mises en œuvre pour réaliser cette fonction. Ce langage fonctionnel est aussi bien pour Jakobson que pour Jakubinskij l'objet primaire d'une investigation scientifique.

On ne peut nier que les auteurs de thèses et Jakubinskij développent un fonctionnalisme dans le domaine de la langue qui est bien différent de celui qui s'est généralisé en linguistique ultérieurement (DUCROT \& SCHAEFFER, 1995, p. 41-48). II s'agit d'un fonctionnalisme qui fait l'économie des concepts de système et de structure et s'avère par-là beaucoup plus descriptif et inductif que son versant plus récent ${ }^{4}$. Ce constat se trouve confirmé par Jakubinskij qui développe tout au début de son livre l'idée que la langue n'est un objet d'analyses linguistiques qu'en tant qu'objet réel. Aborder le langage comme un phénomène qui est immédiatement donné dans la perception va à l'encontre de toute pensée structuraliste qui identifie l'objet de la linguistique avec un objet construit, ou en d'autres termes avec un produit de la connaissance (c'est le point de vue qui crée l'objet). Dans les Thèses du C.L.P., Jakobson souligne également le caractère réel de l'objet de la linguistique en disant:

L'étude d'une langue exige que l'on tienne rigoureusement compte de la variété des fonctions linguistiques et de leurs modes de réalisation dans le cas considéré. Lorsqu'elle n'en tient pas compte, la caractérisation, soit synchronique, soit diachronique, d'une langue quelconque s'en trouve nécessairement déformée et, jusqu'à un certain point, fictive. (THESES, 1929, p. 14).

Le caractère inductif, même empirique, de la linguistique telle qu'elle est préconisée par Jakobson et Jakubinskij renvoie à une compréhension du langage

\footnotetext{
${ }^{4}$ P. Seriot (1999) propose une enquête sur l'apparition de la notion de structure chez les représentants russes du cercle linguistique de Prague et montre la différence dans la compréhension et dans l'utilisation de ce terme par rapport à de Saussure. Une telle analyse serait à refaire pour clarifier le terme de fonction tel qu'il est utilisé par les Russes.
} 
intérieur extrêmement intéressante pour notre discussion. On dira que le langage intérieur est une des formes de la diversité fonctionnelle de la parole. En suivant cette logique, Jakobson commence dans les thèses la classification des langues de diverses fonctions par la distinction entre langage intérieur et langage manifesté :

Ce dernier n'est, pour la majorité des sujets parlants, qu'un cas particulier, car ils emploient les formes linguistiques en pensant plus souvent qu'en parlant: aussi est-il erroné de généraliser et surestimer l'importance, pour la langue, de l'aspect phonique tout extérieur, et faut-il tenir compte des faits potentiels linguistiques. (THESES, 1929, p. 14).

Cet énoncé est d'une importance non négligeable pour la manière dont le langage intérieur a été discuté dans la tradition russe/soviétique. Retenons ici que Jakobson procède à une forte relativisation du rôle du langage extériorisé en affirmant que le langage intérieur est beaucoup plus utilisé dans les pratiques langagières de tous les jours que le langage extériorisé, car on pense plus souvent avec la langue que I'on ne parle. Il semble donc que le Jakobson de 1929 soutient une idée bien opposée à celle qu'il propose en 1954 (voir p. 3 de ce texte). Mais ce n'est pas le parcours intellectuel de Jakobson qui nous intéresse ici. Ce qui nous intéresse est le fait que Vygotskij emprunte à Jakubinskij une idée également développée par les auteurs des thèses. II s'agit de la considération du langage intérieur comme un langage au sens strict du mot, à droit égal avec le langage extériorisé et communicatif et en conséquence non inférable de celui-ci. Le résultat d'une telle approche est qu'on peut découvrir des faits linguistiques spécifiques au langage intérieur et jusqu'à présent pas considérés comme tels, ce qui relativise également le rôle accordé habituellement à certains faits (comme l'aspect phonique) dans l'analyse de la langue. Nous appelons semiotico-descriptive l'étude du langage intérieur dans cette perspective. II reste cependant à signaler que nous ne trouvons ni chez Jakobson ni chez Jakubinskij une véritable analyse linguistique du langage intérieur ; c'est Vygotskij qui propose, dans le septième chapitre, une description détaillée de sa structure.

\section{Une description linguistique du langage intérieur}


Les traits linguistiques les plus importants que Vygotskij assigne à cette forme de la langue concernent aussi bien l'aspect syntaxique que l'aspect sémantique de l'énoncé. L'auteur parle d'une simplification syntaxique maximale, une condensation absolve de la pensée, qui au fond ne signifie rien d'autre qu'une élimination complète de la syntaxe du langage oral et une structure purement prédicative des propositions. La tendance au caractère prédicatif de la syntaxe - propre au langage intérieur - est accompagnée d'une réduction des éléments phonétiques. Il y a en général dans le langage intérieur un rapport entre aspect sémantique et aspect phonétique tout à fait différent de celui qui existe dans le langage extériorisé. Aussi bien Jakobson que Jakubinskij et Vygotskij relativisent suite à cette observation l'importance des éléments phonétiques pour une analyse du langage en général et mettent pour l'analyse du langage intérieur la signification du mot au premier plan :

Le langage intérieur utilise de préférence l'aspect sémantique du langage et non son aspect phonétique. Cette relative indépendance de la signification du mot à l'égard de son aspect phonétique se manifeste dans le langage intérieur avec une netteté extrême. (VYGOTSKIJ, 1997, p. 480)

II s'ensuit que la structure sémantique du langage intérieur présente quelques spécificités: Vygotskij parle de prépondérance du sens sur la signification, ce qui explique le fait que les mots peuvent changer de sens ou qu'ils continuent d'exister même si le sens s'évapore. De là découlent deux autres particularités sémantiques: l'agglutination des mots et la création d'idiotismes (ibid. p. 487), tous deux intraduisibles dans le langage extériorisé, ce qui, ainsi que l'affirme a-t-il pu l'auteur, entraîne "une inintelligibilité du langage égocentrique ou intérieur, que tous les observateurs ont relevée" (ibid. p. 485).

Mais comment Vygotskij a-t-il pu établir cette description du langage intérieur qui de fait n'est pas accessible à un observateur? A part des analyses expérimentales sur le langage égocentrique chez l'enfant qui prennent appui sur les recherches faites dans ce domaine par Piaget ${ }^{5}$, Vygotskij recourt aux comparaisons avec des

\footnotetext{
5 “Lors de l'étude de processus internes complexes, on doit, pour soumettre à l'expérience, pour objectiver le processus interne que l'on observe, reconstituer expérimentalement son aspect extérieur en le rattachant à une activité extérieure quelconque, on doit l'amener à s'extérioriser afin de pouvoir procéder à son analyse fonctionnelle objective, fondée sur les observations de l'aspect extérieur du
} 
phénomènes analogues dans le langage oral qu'il trouve dans la littérature et dans le livre de Jakubinskij. Ainsi, il cite deux dialogues célèbres pour appuyer et illustrer ses descriptions. Le premier a été décrit par Dostoevskij et se déroule entre six ouvriers ivres qui, en rentrant chez eux, communiquent par l'énoncé d'un seul substantif ne figurant pas dans le lexique officiel; pourtant, les six arrivent à se comprendre parfaitement (ibid. p. 470-471). Le deuxième dialogue est celui entre Levin et Kitty relaté dans Anna Karenine par Tolstoj, dans lequel les deux amoureux échangent exclusivement à l'aide des initiales des mots. Dans les deux cas, le langage utilisé par les interlocuteurs possède des caractéristiques semblables au langage intérieur comme la prédicatisation ou l'abréviation. En règle générale, cette utilisation très spéciale du langage extériorisé suppose deux conditions indispensables pour la réussite de la communication : d'une part, les interlocuteurs doivent avoir présent à l'esprit le même sujet du jugement - ce dont on parle dans l'énoncé. Autrement dit, une étroite communion psychologique doit exister entre eux, une espèce de communauté d'aperception; d'autre part le contenu intime de la pensée est transmis par l'intonation. L'intonation est la deuxième source d'une compréhension correcte lorsqu'on utilise un langage extériorisé qui a, comme le langage intérieur, une tendance au caractère prédicatif. Vygotskij conclut :

[...] ces mêmes circonstances grâce auxquelles des jugements purement prédicatifs sont parfois possibles dans le langage oral et qui font totalement défaut dans le cas du langage écrit se présentent constamment et invariablement lorsqu'il y a langage intérieur, elles sont inséparables de celui-ci. (ibid. p. 474)

Le souci de Vygotskij de rendre objectif un processus qui se déroule exclusivement à l'intérieur du locuteur et qui tient de là sa spécificité implique une finalité épistémologique qui dépasse le cadre d'une recherche purement linguistique. On pourrait la dégager à travers ses réflexions et ses critiques faites à propos d'un autre courant théorique bien connu en ce temps-là, à savoir la psychologie de la pensée de l'école de Würzburg. L'intérêt pour ce courant a été éveillé chez Vygotskij par leur démarche qui tente d'expliquer la pensée à partir de l'analyse du processus de

processus interne. Par contre, avec le langage égocentrique nous avons affaire en quelque sorte à une expérimentation naturelle de ce type" (Vygotskij, 1997, p. 444-445).

Eutomia, Recife, 19 (1): 79-99, Jul. 2017 
la pensée tel qu'il se déroule dans la tête du sujet parlant. Vygotskij s'est senti interpellé par le débat entre Wundt et l'école de Würzburg dans lequel il prend sans hésitation la position de la psychologie de la pensée. Tandis que Wundt traitait les fonctions psychiques supérieures exclusivement par l'intermédiaire d'une analyse des produits de la pensée, les chercheurs autour de Külpe analysaient les processus de la pensée qui se déroulaient immédiatement sous leurs yeux. Pour Wundt, la pensée n'est analysable que dans les produits historiques, dans la langue ou les coutumes, dans la psychologie d'un peuple. En suivant son raisonnement, il faudrait admettre que l'accès à la pensée n'est possible qu'à travers des formes cristallisées, inertes et fixes. Cette opinion n'a pas été partagée par l'école de Würzburg. Leurs membres traitaient la pensée comme un processus dynamique, comme étant dans le temps, comme déroulement réel ou encore comme activité productrice.

Si Vygotskij s'impose la même tâche que la psychologie de la pensée, il formule néanmoins une critique vis-à-vis de l'école de Würzburg concernant l'indépendance absolve de la pensée à l'égard du mot, telle qu'elle est affirmée par ce courant

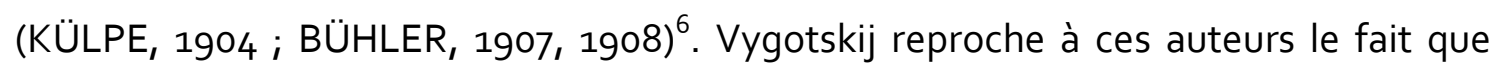
dans leur perspective le mot n'est rien d'autre qu'une manifestation extérieure de la pensée, son vêtement, il ne prend aucunement part à sa vie intérieure. Afin d'éviter que l'associationnisme dominant dans la psychologie empirique du début du siècle et fortement critiqué par l'école de Würzburg en ce qui concerne l'appréhension de la pensée reste intact dans le domaine de la langue, Vygotskij affirme que la nature de la liaison entre le mot et la signification peut se modifier. D'ailleurs, c'est notamment une telle modification qu'il croit à la source du développement de la pensée conceptuelle dans l'ontogenèse de l'enfant. Les analyses entamées par l'auteur dans ce domaine ont clairement montré que la signification d'un mot se développe. En utilisant les mots, l'enfant construit en fonction de son âge des concepts à caractère différent; avant qu'il devienne capable d'élaborer un concept scientifique au sens strict du mot il construit des complexes associatifs, complexes-collection, complexes en chaîne, pseudo-concepts etc. (VYGOTSKIJ, 1997, chap. V). La conclusion principale

\footnotetext{
${ }^{6}$ Dans ses travaux ultérieurs, Bühler change cette perspective et attribue au langage un rôle beaucoup plus important dans la discussion des phénomènes psychiques (Bühler 1982 [1934] : une traduction de ce texte en français est en cours).

Cf. a referência da tradução, Bühler, 2009 (literatura atualizada).
} 
que Vygotskij en tire atteste que la signification d'un mot n'est pas immuable, elle varie avec les différents modes de fonctionnement de la pensée.

Suite à l'analyse des traits linguistiques du langage intérieur on pourrait alors dire que ce qu'on observe dans l'ontogenèse de l'enfant - un développement de l'aspect sémantique du mot - constitue la caractéristique principale du langage intérieur. Dans le langage intérieur le rôle fonctionnel de la signification du mot dans I'acte de la pensée se montre d'une manière exemplaire. Ce constat est plus important qu'il y paraît, car il force à nous interroger sur la manière dont la pensée se réalise et nous renvoie en conséquence directement à la personne qui pense. Pour préciser cette nouvelle perspective qui découle des descriptions linguistiques du langage intérieur, nous nous référons à un autre exemple emprunté cette fois-ci par Vygotskij à Stanislavskij, grand metteur en scène de théâtre russe. En préparant la présentation de la pièce de Griboedov "Le malheur d'avoir l'esprit", Stanislavskij voulait comprendre la phrase "bienheureux celui qui croit, il a chaud au cœur" adressée par Tchatski à Sofia, dans le sens de "Cessons cette conservation". Vygotskij réplique à Stanislavskij :

Mais nous pourrions à tout aussi juste titre y voir l'expression d'une autre pensée 'Je ne vous crois pas. Vous dites des paroles consolantes pour m'apaiser.' Ou nous pourrions encore lui substituer celui-ci que la phrase pourrait tout aussi bien exprimer : 'Ne voyez-vous pas comme vous me tourmentez. Je voudrais vous croire. Ce serait pour moi la félicité'. (ibid. p. 491).

Vygotskij soulève dans cet exemple trois manières différentes de penser à l'aide d'un même énoncé. Il existe indéniablement d'autres possibilités de penser avec cette phrase, mais comment peut-on décider quelle pensée est actualisée en réalité à travers cette phrase? Que Stanislavskij se décide pour la première attribution peut être expliqué par sa manière de concevoir le personnage de Tchatski et son souci motivé par sa profession de metteur en scène - de donner à ce personnage une certaine cohérence de caractère tout au long du spectacle. Dans la vie hors du théâtre, une telle attribution se heurte à la personne elle-même. Il ne faut pas confondre ici ce qui est pensé avec ce qui est dit et ce qui attend en conséquence une compréhension. Dans l'optique de la compréhension, la phrase énoncée demanderait et justifierait 
l'interprétation (donner du sens) aussi bien par le locuteur que par l'interlocuteur. L'optique de Vygotskij vise cependant non pas la compréhension mais le processus de la pensée même : qu'est-ce qui est pensé au travers de cette phrase ? La discussion du langage intérieur telle qu'elle est proposée par Vygotskij suggère une différence entre ces deux optiques :

Ce cours et ce mouvement de la pensée ne coïncident pas directement avec le déroulement du langage. Les unités de base de la pensée et celles du langage ne coïncident pas. [...] Ils sont liés l'un à l'autre par des transitions complexes, des transformations complexes mais ils ne se recouvrent pas l'un l'autre comme les lignes droites superposées" (ibid. p. 489490).

Et il poursuit une page plus loin: "On voit nettement ici la ligne de démarcation entre la pensée et le mot, Rubicon infranchissable pour le locuteur" (ibid. p. 491).

Au moment où les sciences humaines ont trouvé leur entrée dans l'amphithéâtre des sciences, Vygotskij confirme l'irréductibilité de leur objet à une donnée de la science dans la mesure où il met en lumière avec le langage intérieur une utilisation du langage qui le rend inintelligible pour autrui. Cela ne fait aucun doute, le langage intérieur n'est intelligible pour autrui que dans des conditions exceptionnelles, dans les relations comme la communion psychique qui font défaut dans une recherche scientifique. Mais s'arrêter à ce constat signifierait ne pas saisir une idée essentielle dans sa démarche. Vouloir donner une description sémiotique du langage intérieur signifie vouloir saisir la part réelle que le sujet a dans sa propre pensée ${ }^{7}$. Par cette part réelle, nous comprenons la chose suivante. La pensée qui se fait à travers le langage intérieur n'est pas identique à ce qui est objectivable et réellement objectivé dans le passage du langage intérieur vers un langage extériorisé. Dans le langage intérieur la pensée émerge, se constitue et ce moment de constitution est inséparable du sujet. La démonstration de Vygotskij est convaincante; il voit dans le fait que le sujet utilise les significations du mot d'une manière fonctionnelle le fondement et la réalité du langage intérieur. Cela veut dire

\footnotetext{
${ }^{7}$ Vygotskij développe un concept du réalisme dans le cadre d'une théorie de connaissance de la psychologie qui étaye plus en détail cette idée, voir Vygotskij 1999 [1927] : 48-49, 269-274.
} 
qu'on ne peut pas localiser la pensée dans le foyer intérieur du sujet, car c'est en utilisant le langage que le sujet donne naissance à la pensée. A travers le langage intérieur, la pensée émerge en se détachant du foyer intérieur du sujet, elle ne peut alors pas être inférée ni des intentions ni des affects compris comme des phénomènes antérieurs à ce procès. Dans ce sens, le langage intérieur a une certaine réalité pour ce sujet bien que cette réalité soit crée par lui. Cette idée de la part réelle du sujet qui devrait faire l'objet des recherches en sciences humaines peut être dégagée chez d'autres auteurs comme par exemple le sociologue Alfred Schütz. Cet auteur désigne le processus de l'action en cours de déroulement comme " temps interne " (SCHÜTZ, 1998, p. 74-76) et théorise avec quasiment le même adjectif que Vygotskij l'irréductibilité de la part du sujet dans l'action à ce qui est objectivable à travers des interprétations de l'action développées après coup soit par le sujet soit par les autres. En outre, I'adjectif " interne " signale pour Schütz la liaison inséparable entre le sujet et son action, qui trouve son fondement et sa réalité dans le cours du déroulement de l'action (FRIEDRICH, 2001).

La proposition d'analyser le langage intérieur pas seulement d'un point de vue psycho-génétique mais de le soumettre à une analyse sémiotico-descriptive a des retombées importantes pour les débats épistémologiques au sein des disciplines qui se constituent à cette époque comme sciences à droit égal avec les sciences de la nature. Au lieu de rechercher un type de connaissance adapté aux sciences humaines et de s'inscrire dans le débat bien familier autour de l'explication et de la compréhension, Vygotskij pose la question de la réalité spécifique des objets de ce groupe de sciences. Dans cette perspective, ses réflexions sur le langage intérieur concernent plus qu'un domaine de la recherche linguistique méprisé et négligé. Elles impliquent une compréhension bien précise de ce qui devrait faire l'objet des linguistes et des psychologues, une compréhension qui mérite d'être discutée encore de nos jours.

\section{Références bibliographiques}


ARCHAIMBAULT, S. Un texte fondateur pour l'étude du dialogue: De la parole dialogale (L. Jakubinskij). In: Histoire, Epistémologie, Langage 22/1 : 2000, pp. 99-115.

BAKHTINE, M. (Volochinov, Valentin N.) Le marxisme et la philosophie du langage: Essai d'application de la méthode sociologique en linguistique. Paris: Minuit, 1977 [1929].

BÜHLER, K. Tatsachen und Probleme zu einer Psychologie der Denkvorgänge. I.

Über Gedanken. In: Archiv für die gesamte Psychologie, 9. 1907.

Tatsachen und Probleme zu einer Psychologie der Denkvorgänge. II. Über Gedankenzusammnehänge. III. Gedankenerinnerungen. In: Archiv für die gesamte Psychologie, 12. 1908.

Sprachtheorie. Die Darstellungsfunktion der Sprache. Stuttgart-New-York: Fischer, 1982 [1934].

Théorie du langage. La fonction représentationnelle. Édité par D. Samain et J.

Friedrich, préface de Jacques Bouveresse. Marseille: Agone, 2009.

DORON, R. ; PAROT, F. Dictionnaire de psychologie. Paris : Presses universitaires de France, 1998.

DUCROT, O.; SCHAEFFER, J-M. Nouveau dictionnaire encyclopédique des sciences du langage. Paris: Sevil, 1995.

FEHR, J. Saussure entre linguistique et sémiologie. Paris: PUF, 2000.

FODOR, J. A. The Language of Thought. Cambridge Mass.: Harvard Univ. Press, 1975.

FONTAINE, J. Le cercle linguistique de Prague. Tours: Mame, 1974.

FRIEDRICH, J. Der Gehalt der Sprachform. Paradigmen von Bachtin bis Vygotskij. Berlin: Akademie-Verlag, 1993.

Quelques réflexions sur le caractère énigmatique de l'action. In : J.-M. Baudouin \& J. Friedrich (Ed.), Théories de l'action et éducation (Raisons éducatives, Nº 4, pp. 93-112).

Bruxelles : DeBoeck. 2001.

. L'idée des instruments médiatisants. Un dialogue fictif entre Bühler et Vygotski. In:

Y. Clot (Ed.), Vygotski maintenant (pp. 255-270). Paris : La Dispute. 2012.

The use and function of the notion of dialogue in the Soviet-Russian discourse of the 1920s, especially with Yakubinski and Vygotsky. In: M.-C. Bertau (Ed.), Think about language dialogically - Understand action dialogically, Interdisciplinary conference, summer 2005. (http://epub.ub.uni-muenchen.de/2020/1/Jakubinskij_en.pdf) 2006. 
Die Apperzeptionsgebundenheit des Sprechens. Ein historischer Exkurs in die Diskussion um die innere Sprache. In: M.-C. Bertau, A. Werani \& G. Kegel ( Hg.), Psycholinguistische Studien 2 (pp. 27-59). Aachen: Shaker. 2005.

L'idée d'instrument psychologique chez Vygotski. Revista Italiana di Filosofia del Linguaggio : Vygotskij et il linguaggio (éd. par F. Cimatti, L. Mecacci, E. Velmezova), vol. 6, $n^{\circ}$ 2, 2012, p. 189-201. <http://www.rifl.unical.it/index.php/component/content/article/182vygotsky-and-language-indice.html>

. Vygotsky's idea of psychological tools. In: A. Yasnitsky, R. van der Veer \& M. Ferrari (Ed.), The Cambridge Handbook of Cultural-Historical Psychology (pp. 47-62). Cambridge: Cambridge University Press, 2014.

JAKOBSON, R. Novešaja russkaja poezija. Nabrosok pervyi : Podstupy k Chlebnikovu. In: Roman JAKOBSON, R. Selected Writings. Vol. 5, 1979.The Hague-Paris-New York: Mouton Publishers, 1921, p. 299-354.

Essai de linguistique générale 1. Paris: Minuit, 1963.

JAKUBINSKIJ, L. P. O dialogičeskoj reči . In JAKUBINSKIJ, L.P. Izbrannye raboty: Jazyk et ego funkzionirovanie. Moskva: Nauka, 1986 [1923], p. 17-58.

KNOBLOCH, C. Sprachpsychologie: ein Beitrag zur Problemgeschichte und Theoriebildung. Tübingen: M. Niemeyer, 1984.

KÜLPE, O. Versuche über Abstraktion. In: Bericht über den 1. Kongress für experimentelle Psychologie in Giessen, Leipzig, 1904.

LURIJA, A. R. Sprache und Bewusstsein. Köln: Pahl-Rugenstein, 1982.

PANACCIO, C. Le discours intérieur de Platon à Guillaume d'Ockham. Paris: Seuil, 1999.

ROCHEX, J-Y. L'oeuvre de Vygotski: fondements pour une psychologie historicoculturelle. In : Revue Française de Pédagogie 120, 1997, p. 105-147.

SAUSSURE, F. Cours de linguistique générale. Paris: Payot, 1983.

SCHÜTZ, A. Choisir parmi des projets d'action. In: SCHÜTZ, A. Eléments de sociologie phénoménologique. Paris : L'Harmattan, 1998 [1951], p. 58-87.

SERIOT, P. Structure et totalité. Les origines intellectuelles du structuralisme en Europe centrale et orientale. Paris: PUF, 1999.

SOKOLOV, A. N. Inner speech and thought, New York-London: Plenum Press, 1972 [1962].

THESES DU CERCLE LINGUISTIQUE DE PRAGUE. In : Travaux du Cercle linguistique de Eutomia, Recife, 19 (1): 79-99, Jul. 2017 
Prague 1, Prague: 1929, pp. 7-29.

VYGOTSKY, L. S. La signification historique de la crise en psychologie. Édition préparée et présentée par Jean-Paul BRONCKART, J-P. et FRIEDRICH, J. Lausanne-Paris: Delachaux et Niestlé, 1999 [1927].

VYGOTSKI, L. S. Pensée et langage. Paris: La Dispute, 1997 [1934].

WERTSCH, J. Vygotsky and the Social Formation of Mind. Cambridge Mass.-London: Harvard University Press, 1985.

\footnotetext{
i Janette Friedrich, Université de Genève, Faculté de psychologie et des sciences de l'éducation (FPSE) Desde 2006, professora - pesquisadora no Laboratoire d'histoire des théories linguistiques do CNRS, Paris. Desde 2013, Membro do grupo de pesquisa "Langage, Dialogue, Interaction (LDI) » da equipe Psychologie du travail et clinique de l'activité dirigée par Yves Clot, CNAM, Paris.

Desde 2015, Colaboradora da equipe de pesquisa "Meaning and Intentionality in Anton Marty ", coordenada por L. Cesalli, departamento de filosofia da Université de Genève.

Janette.Friedrich@unige.ch
} 